\title{
Mortality after cancer among patients with diabetes mellitus: effect of diabetes duration and treatment: (questionable) classification of diabetic patients based on combination of specific glucose-lowering drugs. Reply to Holden SE, Bannister CA, Currie CJ [letter]
}

\author{
Kristina Ranc • Marit E. Jørgensen • Søren Friis • \\ Bendix Carstensen
}

Received: 4 June 2014 / Accepted: 27 June 2014 / Published online: 18 July 2014

(C) Springer-Verlag Berlin Heidelberg 2014

Keywords Cancer · Diabetes duration · Insulin · Mortality · Oral hypoglycaemic agent · Type 2 diabetes

\section{Abbreviation \\ GLP-1 Glucagon-like peptide 1}

To the Editor: In the light of the general comments made about our article [1] by Holden et al [2], we acknowledge that we did not specify with sufficient emphasis that our study was based on the entire population of cancer patients in Denmark, that is to say, it was essentially demographic in its nature.

The uncertainty about the underlying study base has led Holden et al to raise concerns about the lack of characterisation of the cohorts. Unlike clinical observational studies of patients from clinical databases, there is only limited clinical information on the general population of all cancer patients in Denmark, and hence it is not possible to provide a detailed clinical characterisation of the population. On the other hand, our study gives an overall picture of how the entire population of cancer patients fare in relation to their diabetes status.

K. Ranc $\cdot$ M. E. Jørgensen $\cdot$ B. Carstensen

Steno Diabetes Center, Gentofte, Denmark

K. Ranc $(\bowtie) \cdot B$. Carstensen

Section of Biostatistics, University of Copenhagen,

Øster Farimagsgade 5, 1014 Copenhagen, Denmark

e-mail: ksrc@sund.ku.dk

S. Friis

Danish Cancer Institute Research Center, Danish Cancer Society,

Copenhagen, Denmark
The following are our replies to the specific questions raised by Holden et al:

- The classification of patients is unequivocal: '...patients with diabetes but no history of glucose-lowering medication (DM NoMed; $n=15,453$ ), patients exclusively treated with oral hypoglycaemic agents (OHAs) (DM+OHA; $n=17,708)$, or patients on insulin (DM+Ins; $n=9,044)$ ' [1]. The patients receiving glucagon-like peptide 1 (GLP-1) are included in the OHA group; however, the number of cancer patients treated with GLP-1 is negligible. The demographic nature of the study precluded further subdivision of treatment groups.

- In our paper we stated 'Cox models were fitted using time since cancer diagnosis as time-scale using only a fixed HR for each diabetes group compared with No DM'. Thus, we are somewhat puzzled by the impression of Holden et al that the Cox model was only adjusted for age, calendar time and diabetes status when we stated it was adjusted for diabetes status and time since cancer diagnosis.

- Our comment that cancer treatment might differ according to prevalence and severity of diabetes was not meant as a confirmatory statement, but merely speculation. Because of the demographic nature of our study we had no detailed clinical, let alone treatment, information for the available patients.

- Holden et al queried 'Could grouping of the patients, which essentially defines the position in the diabetes treatment pathway, be acting as a proxy for diabetes duration?' In our paper, we explained that the models included smooth terms for interaction between diabetes duration and status, and used separate duration effects in the three groups, so each group was separately controlled for 
diabetes duration. Therefore, the statement in the results section that 'diabetes duration at cancer diagnosis did not influence the mortality estimates' should have been qualified by making it clear that this statement referred to all three duration estimates as seen in Fig. 4 of our paper.

- Holden et al also state 'A further analysis including only those people with a duration of diabetes of 2 years at cancer diagnosis was carried out, but the reason for using this arbitrary cut-off was not explained.' Actually, no such analysis was performed, but it is possible that Holden et al misinterpreted the (admittedly arbitrary) choice of 2 years of diabetes duration in the estimation of mortality rate ratios as a function of time since cancer diagnosis. When there is interaction both between time since cancer diagnosis and group and between diabetes duration and group, one interaction (Fig. 3) can only be reported by fixing a reference point for the other (Fig. 4), in this case at 2 years of diabetes duration. We might have stated this general feature of traditional epidemiological modelling more clearly.

- Holden et al mention that it would have been useful to report the number in each group. We would like to point out that these numbers are actually presented in Table 1 of our paper.

- According to Holden et al, "the patterns of modelled data would be more persuasive if the authors had provided actual survival data to better understand how the unusual biological patterns of survival from their statistical models had been distilled'. We did indeed provide survival data, but instead of traditional survival analysis, we used Poisson modelling as the primary tool for our proportional hazards analysis. The rationale behind this choice was the assumption that continuously varying mortality rates are more biologically plausible than allowing an arbitrary form of mortality as function of time, as is often seen when authors report survival curves from a Cox model. It can be argued that we should have provided a detailed exposition of the relative merits and suitability of the various approaches, but we considered that such detailed statistical elaboration was not suitable for the format of Diabetologia. The interested reader may find an overview of this discussion in a preprint [3].
- Holden et al quote a study on the occurrence of cancer [4], which reported a higher cancer occurrence among sulphonylurea users than among metformin users. It is difficult to perceive how that study, based on a subset of diabetic patients, should have any direct relevance to survival following cancer diagnosis in the general Danish population.

- We agree with Holden et al that there is nothing in this study that precludes or supports an adverse effect of insulin treatment on cancer survival per se.

- Although we regarded the paper as a survival study of cancer patients, we should have reported that B. Carstensen and M. E. Jørgensen are employees of Steno Diabetes Center, a research institution and hospital owned by NovoNordisk, and both are stockholders of NovoNordisk. S. Friis is an employee of the Danish Cancer Society.

Duality of interest The authors declare that there is no duality of interest associated with this manuscript.

Contribution statement All authors were responsible for the conception and design of the manuscript, drafting the response letter and revising it critically for important intellectual content. All authors approved the version to be published.

\section{References}

1. Ranc K, Jørgensen ME, Friis S, Carstensen B (2014) Mortality after cancer among patients with diabetes mellitus: effect of diabetes duration and treatment. Diabetologia 57:927-934

2. Holden SE, Bannister CA, Currie CJ (2014) Mortality after cancer among patients with diabetes mellitus: effect of diabetes duration and treatment: (questionable) classification of diabetic patients based on combination of specific glucose-lowering drugs. Diabetologia 57. doi: 10.1007/s00125-014-3281-1

3. Carstensen B (2006) Demography and epidemiology: practical use of the Lexis diagram in the computer age. Or: who needs the Cox-model anyway? Technical Report 06.2. Department of Biostatistics, University of Copenhagen. Available from: http://biostat.ku.dk/ reports/2006/rr-06-2.pdf

4. Currie CJ, Poole CD, Gale EA. The influence of glucose-lowering therapies on cancer risk in type 2 diabetes. Diabetologia 52: 1766-1777 